\title{
A Preconcentrator Coupled to a GC/FTMS: Advantages of Self-Chemical Ionization, Mass Measurement Accuracy, and High Mass Resolving Power for GC Applications
}

\author{
Touradj Solouki, Jan E. Szulejko, Justin B. Bennett, and LeRae B. Graham \\ Department of Chemistry, University of Maine, Orono, Maine, USA
}

\begin{abstract}
Coupling of a cryogenic preconcentrator (PC) to a gas chromatograph/Fourier transform ion cyclotron resonance mass spectrometer (GC/FT-ICR MS) is reported. To demonstrate the analytical capabilities of the PC/GC/FT-ICR MS, headspace samples containing volatile organic compounds (VOCs) emitted from detached pine tree twigs were analyzed. Sub-ppm mass measurement accuracy (MMA) for highly resolved $\left(\mathrm{m} / \Delta \mathrm{m}_{50 \%}>150 \mathrm{k}\right)$ terpene ions was achieved. Direct PC/GC/FT-ICR MS analyses revealed that detached twigs from pine trees emit acetone, camphor, and four detectable hydrocarbon isomers with $\mathrm{C}_{10} \mathrm{H}_{16}$ empirical formula. The unknown analytes were identified based on accurate mass measurement and their mass spectral appearances. Authentic samples were used to confirm initially unknown identifications. Self-chemical-ionization (SCI) reactions furnished an additional dimension for rapid isomer differentiation of GC eluents in real time. (J Am Soc Mass Spectrom 2004, 15, 1191-1200) (C 2004 American Society for Mass Spectrometry
\end{abstract}

$\mathrm{B}$ iomarker analyses are important in many areas of modern analytical science [1]. For example, various chromatographic and mass spectral studies were used to resolve and identify specific biomarkers in diverse fields such as human physiology, forensics, bacteria, and food spoilage [2-6]. FT-ICR MS offers several advantages for characterizing multicomponent mixtures. For example, the high mass measurement accuracy of FT-ICR MS [7-9] limits the number of possible chemical formulas corresponding to an experimentally determined mass value [10].

Recently, we described potential applications of GC / FT-ICR MS to analyze complex sample mixtures such as automobile gasoline [11]. The GC/FT-ICR MS utilizes the separation capability of a conventional GC [12] as well as mass measurement accuracy $[8,9,13,14]$ and superior mass resolving power of FT-ICR MS [15, 16]. The advantages of multidimensional and/or "hyphenated" chromatography techniques with spectroscopic detectors were reviewed extensively [17].

Often, "real world" biological and environmental samples are complex mixtures, and their complete characterization requires numerous stages of sample preparation or sophisticated data analyses [4]. Our goal is to develop methodologies aimed at detecting low

Published online July 1, 2004

Address reprint requests to Dr. T. Solouki, Department of Chemistry, University of Maine, 5706 Aubert Hall, Orono, ME 04469, USA. E-mail: solouki@maine.edu levels of VOCs that are emitted into the immediate environment by biota [18]. The low levels of VOC analytes at the ppm level (vol/vol) or lower may preclude direct analysis of, for example, human exhaled breath (HEB) with capillary column GC/MS systems [18]. Methods must be developed to separate and/or preconcentrate the VOCs of interest from the bulk components prior to GC/MS introduction. One such method is front-end GC cryogenic microscale purge and trap [18, 19]. Back-end GC cryofocusing has been used for trace FT-ICR mass spectral analysis by Gross and co-workers [20]. We are interested in developing methodologies that require minimal sample preparation and afford improved selectivity for analytical characterization of sampleprints. To realize our objective, we have coupled a preconcentrator (PC) to a GC/FTICR MS (PC/GC/FT-ICR MS) and evaluated its utility. Here, we describe the first PC/GC/FT-ICR MS data from direct headspace analysis of arbor emissions.

A wide range of plants and plant-derived material contain terpenes. It was suggested that terpene compositions may be used to determine the tree types [21, 22] and locations [23, 24], as well as the extent of injuries, such as montane yellowing [25] and forest damage [26], caused by air pollution. Plants may also respond to herbivore feeding and mechanical wounding by releasing targeted chemicals such as proteinase inhibitors [27]. Biogenically emitted monoterpenes may play an important role in atmospheric chemistry of gaseous nitrate $\left(\mathrm{NO}_{3}\right)$ radicals [28]. Hence, accurate measure- 
ment of the tree monoterpenes is important. However, difficulties with handling unresolved complex data [26], lack of an absolute reference for the terpene yield and/or sampling errors, uncertainties associated with invasive sample extraction from conifer needles [26, 29-31], and rough handling [32] highlight the analytical importance of non-invasive, reproducible, and rapid sample analysis for plant characterization based on VOC signatures.

Previously, we reported that high mass resolution FT-ICR MS can be used to identify pressure-dependent gas-phase self-chemical ionization (SCI) product ions under GC/FT-ICR MS conditions [11]. Here, we demonstrate that high mass resolving power and ion-trapping capabilities of FT-ICR mass spectrometry can be utilized to differentiate GC eluted $\alpha$-pinene and camphene isomers in real time. Other researchers reported isomer differentiation based on multiphoton ionization and dissociation (MPI/MPD) [33], GC/MPI-FTMS [34], FTMS proton affinity bracketing [35], collision-induced dissociation (CID) [36-38], CID combined with chromatography [39], ion-molecule reactions combined with chromatographic techniques [40], oxygen negative chemical ionization (ONCI) [41], NCI GC [42], charge inversion MS [43], and by metal oxide sensors [44]. Modifying chromatographic parameters such as GC temperature programming or column type that can alter elution time and/or order will not change mass spectral appearances. Hence, independent of the chromatographic retention time, detector differentiation (i.e., mass spectral differentiation based on analyte properties such as proton affinity and ion-molecule reactivities) can be used to distinguish isomers. The advantages of PC/GC/FT-ICR MS in terms of high mass resolving power, sensitivity, mass measurement accuracy, and isomer differentiation are discussed.

\section{Experimental}

\section{Specimen or Sample Collection}

Pine Twigs. Arbitrarily selected $\sim 5-10 \mathrm{~cm}$ long twigs (with needles attached) were detached manually (by the authors) from a branch (at a height of $\sim 1.5 \mathrm{~m}$ ) of a pine tree (Picea rubens) [45] and placed immediately in ZipLoc (Trinity Packaging, Lewistown, PA) bags (1 per bag) for PC/GC/FT-ICR MS analysis. Samples were collected in 2001, during the month of November, when plant monoterpenes are assumed to return to their base low levels; it has been reported that terpene levels increase in May and return to their base levels by September [46]. To re-confirm the emission of VOCs from the same pine tree, additional samples were analyzed in March 2003. The detached pine twig samples were stored at room temperature in a $28 \times 27 \mathrm{~cm}$ sealed ZipLoc bag. Prior to analysis, the end of the PC sampling line was carefully inserted $(\sim 10 \mathrm{~cm})$ through a small opening made in the ZipLoc seal near $(\sim 1-2 \mathrm{~cm})$ the pine needle specimens. The seal was immediately reclosed and the headspace analyzed. Direct PC/GC/ FT-ICR MS analysis of the VOCs in pine tree twigs did not require any sample treatment. The preconcentrator was programmed (event 2 in PC Event Sequence) to collect a $100 \mathrm{~mL}$ sample of headspace volume surrounding the pine needles. Method blanks did not show any acetone or terpenes from the background air or unused ZipLoc bags.

Reagents. All reagents and solvents were purchased from commercial sources and used without further purification. The authentic samples were purchased from Aldrich Chemical Company, Inc. (Milwaukee, WI).

\section{Instrumentation}

The PC/GC/FT-ICR MS consisted of three major components; a 3-stage Entech 7100 series Preconcentrator (PC) (Entech, Simi Valley, CA), an SRI GC system (Las Vegas, NV) and an IonSpec 7-T FT-ICR mass spectrometer (IonSpec Corp., Lake Forest, CA). A schematic of the apparatus is shown in Figure 1. The exact details on, fluid flow (i.e., for $\mathrm{He}$ and $\mathrm{N}_{2}$ gases and $\mathrm{LN}_{2}$ cryogen), multipositional valve assemblies (denoted as V1, V2, and V3) and vacuum lines within the PC apparatus have been either omitted or simplified for illustrative purposes only. The flow rates, temperatures and timing of the preconcentrator event sequence was under computer control (Toshiba, Equium 7100D, Irvine, CA). Detailed description of the preconcentrator operation has been published elsewhere [19]. Glass bead, Tenax (Alltech-Deerfield, IL), and cryofocuser traps were used to remove or reduce common air components (e.g., Ar, $\mathrm{CO}_{2}, \mathrm{H}_{2} \mathrm{O}, \mathrm{N}_{2}, \mathrm{O}_{2}$, etc.) and concentrate the volatile organics. The cryofocused organics were desorbed by flash-heating and injected on a GC column as a concentrated sample plug. The details of coupling a GC to the FT-ICR MS and its potential analytical applications were discussed earlier [11]. The labels SC and TL, in Figure 1, denote sample container and heated transfer line, respectively.

$P C$. The trapping and preconcentration of the VOCs of interest were performed on an Entech 7100 PC using the microscale purge and trap technique, utilizing three trapping modules (labeled M1, M2, and M3 on Figure 1 ). The sequence of events used in our experiments was as follows:

\section{PC Event Sequence:}

1. Prior to use, the PC was leak tested, flushed with nitrogen and baked at appropriate temperatures $\left(\sim 120^{\circ} \mathrm{C}\right.$ for $\mathrm{M} 1, \sim 180{ }^{\circ} \mathrm{C}$ for $\mathrm{M} 2$, and $\sim 70{ }^{\circ} \mathrm{C}$ for cryofocuser).

2. A user defined volume of the head space, or a gas sample (e.g., $100 \mathrm{~mL}$ standard atmosphere for Figure 2) containing trace VOCs, was pumped through the first trap (T1) located in module 1 (M1). In T1, the VOCs and water and some $\mathrm{CO}_{2}$ were condensed 


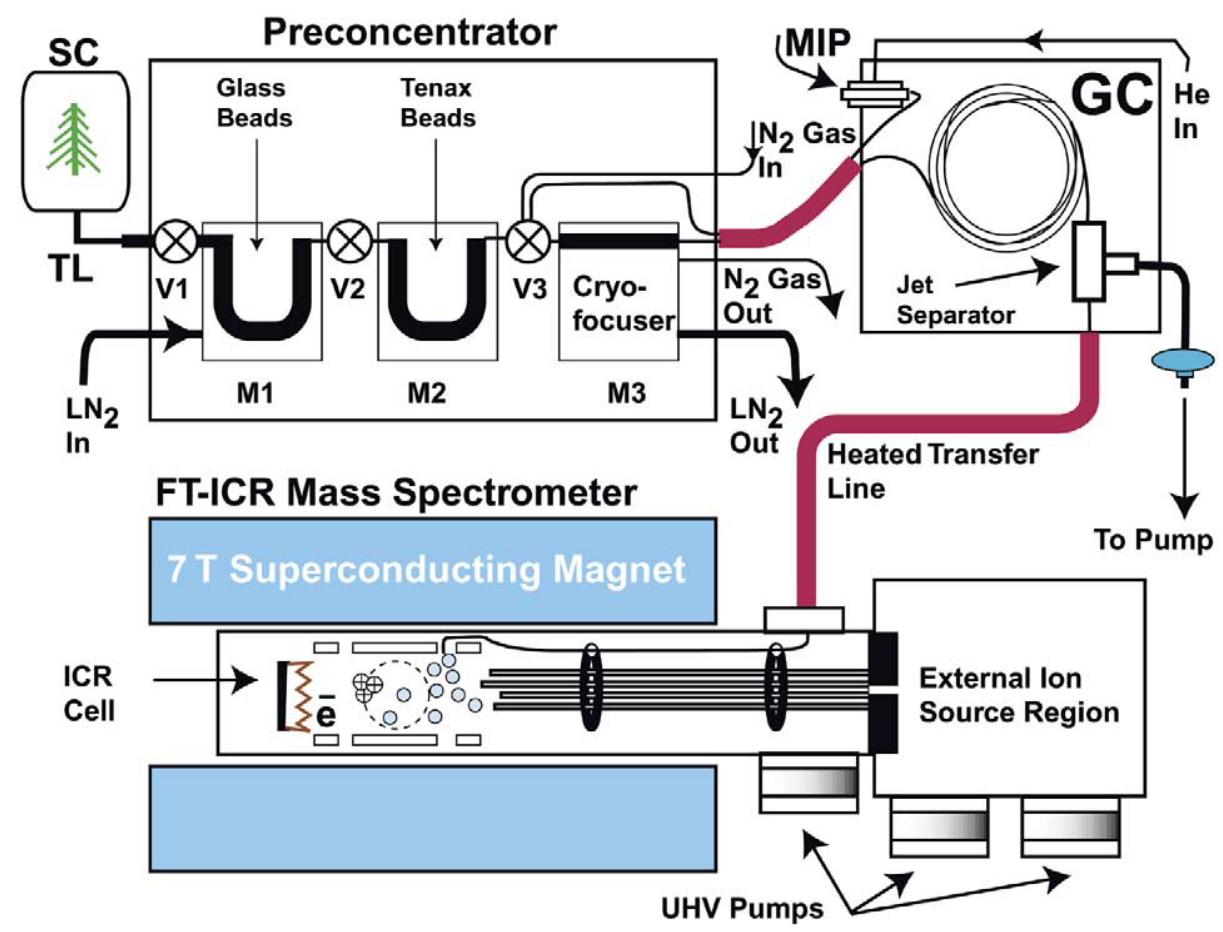

Figure 1. A simplified schematic of the PC/GC/FT-ICR mass spectrometer: The exact gas flow details (i.e., for $\mathrm{He}, \mathrm{N}_{2}$ gases and also for $\mathrm{LN}_{2}$ cryogen flow) and configurations on valve systems contained within the PC apparatus have been either omitted or simplified for illustrative purposes only. Label SC denotes sample container, MIP denotes GC manual injection port and TL stands for heated transfer line.

and absorbed on the glass beads (maintained at $-150{ }^{\circ} \mathrm{C}$ ), whereas the $\mathrm{N}_{2}, \mathrm{O}_{2}$ and Ar were not.

3. $75 \mathrm{~mL}$ of He at standard atmospheric pressure were flushed through $\mathrm{M} 1$ at $-150^{\circ} \mathrm{C}$ over $5 \mathrm{~min}$.

4. The trap (T2) containing Tenax beads in module 2 (M2) was cooled to $-30^{\circ} \mathrm{C}$.

5. M1 was warmed to $\sim 20^{\circ} \mathrm{C}$ (for data shown in Figure 2).

6. $40 \mathrm{~mL}$ of He at standard atmospheric pressure was used to purge T1 to effect the transfer of VOCs into $\mathrm{T} 2$.

7. The cryofocuser module (M3) was cooled to $-160{ }^{\circ} \mathrm{C}$. The actual cryofocuser (CF) consisted of a section of megabore GC guard column that could be heated from $-160{ }^{\circ} \mathrm{C}$ to $\sim 70{ }^{\circ} \mathrm{C}$ (in less than $30 \mathrm{~s}$ ) by passing heated $\mathrm{N}_{2}$ gas over the $\mathrm{CF}$ line.

8. T2 in M2 was heated to $180^{\circ} \mathrm{C}$.

9. Mutlipositional valve assemblies directed the GC carrier gas (He) through $\mathrm{T} 2$ to purge any trapped VOCs into the $\mathrm{CF}$, for $2.5 \mathrm{~min}$.

10. The CF (megabore capillary line) was flash heated to $\sim 70{ }^{\circ} \mathrm{C}$. At this point, the GC temperature program and FT-ICR MS mass spectral acquisition were simultaneously started.

11. Mutlipositional valve assemblies directed the GC carrier gas (He) through the CF to purge any VOCs onto the GC column over a user-defined period. After $1.5 \mathrm{~min}$, the $\mathrm{CF}$ was cooled down to $\sim-120^{\circ} \mathrm{C}$ (the temperature of M3). During the GC run, no further cryogen was introduced into M3 and the ambient warming slowly increased the CF temperature to $\sim-60{ }^{\circ} \mathrm{C}$ in $30 \mathrm{~min}$. The $\mathrm{CF}$ was interfaced to the GC column by means of a heated transfer line (maintained at $100^{\circ} \mathrm{C}$ ) containing a $\sim 1 \mathrm{~m}$ length of deactivated megabore GC guard column. This line conveyed both the carrier gas and the purged VOCs to the GC column.

GC Operation. The purged VOC sample (at event 11 in PC Event Sequence) was injected onto a $60-\mathrm{m}(0.28 \mathrm{~mm}$ i.d., $3 \mu \mathrm{m}$ crossbonded $100 \%$ dimethyl polysiloxane stationary phase coating) MXT-1 capillary column (Restek Corporation, Bellefonte, PA) housed in an SRI model 8610C GC oven. The effluent exiting the capillary column was directed into a SGE glass molecular jet separator (SGE Inc., Austin, TX). Both the GC column and molecular jet separator were located within the GC oven [11]. The helium and analyte of interest were transferred from the molecular jet separator to the internal ion source of the FT-ICR MS [11] by means of a 2-m length, $0.5 \mathrm{~mm}$ i.d., MXT guard capillary column (Restek Corporation, Bellefonte, PA).

The section of the transfer line ( $\sim 1 \mathrm{~m}$ in length) external to the FT-ICR MS ultrahigh vacuum (UHV) housing, was heatable up to $200^{\circ} \mathrm{C}$. However, the transfer line within the UHV housing was unheated. The transfer line entered the UHV housing by means of a 1/8-in swage fitting. The terminus of the transfer line 


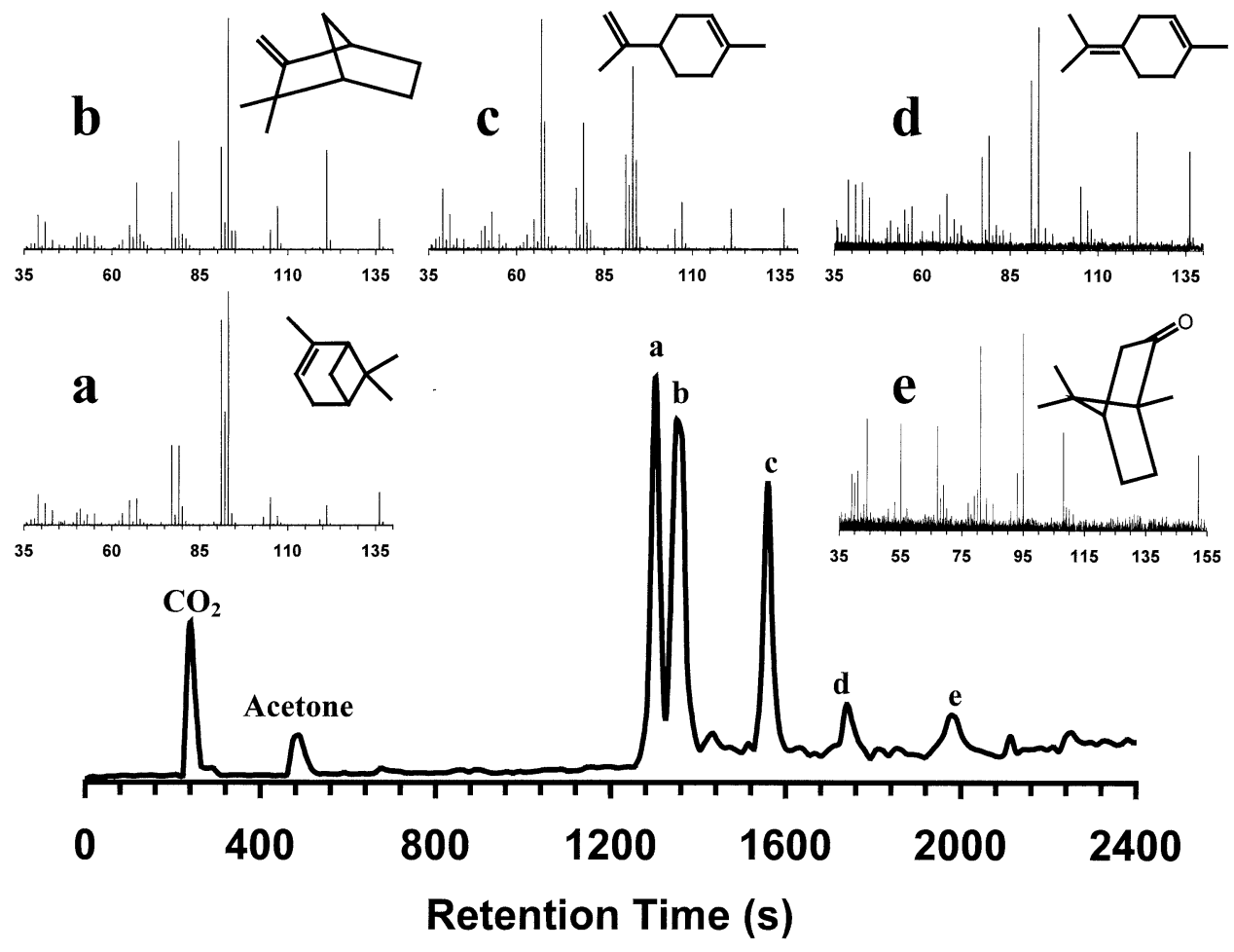

Figure 2. The TIC from a $100 \mathrm{~mL}$ sample of headspace volume surrounding pine twigs at room temperature in a large volume sealed ZipLoc bag: arbitrarily selected $(\sim 5 \mathrm{~cm}$ long) twigs containing pine needles had been detached manually from a Picea rubens tree situated in the environs of the university campus for headspace analysis. Insets (a to e) show the mass spectra for TIC peaks labeled a to e. VOC assignments ( $\alpha$-pinene, camphene, limonene, terpinolene, and camphor, in order of elution time) are shown as structures on each mass spectral inset.

was directed towards the center of the ICR cell through a gap separating adjacent transmitter and receiver plates of the ICR cell. To acquire the high resolution mass spectral data in Figure 5 and Table 1, the jet separator was removed and a pulsed valve interface (similar to the design of Sack and Gross [47]) was inserted in the transfer line between the GC and FT-ICR vacuum housing.

GC temperature programming and column head pressure. A user-defined GC temperature program was used to acquire the data presented in Figures 2, 3, and 5. The GC parameters, including temperature programming and column head pressure, for all three experiments presented in Figures 2, 3, and 4 were identical. The GC injection port was kept at $200{ }^{\circ} \mathrm{C}$, and samples were PC(Figure 2) or manual-injected (Figures 3, 4, and 5) onto the column. The following GC temperature program, at a column-head pressure of $15 \mathrm{psi} \mathrm{He}$, was used for headspace analysis of pine twigs (Figures 2, 3, and 4): $40^{\circ} \mathrm{C}$ for $3 \mathrm{~min}$, ramp at $20^{\circ} \mathrm{C}$ per min to $140{ }^{\circ} \mathrm{C}$, ramp at $2{ }^{\circ} \mathrm{C}$ per min to $220^{\circ} \mathrm{C}$ and then hold at $220^{\circ} \mathrm{C}$ for two min. To reduce the GC retention-time, for high mass resolution studies of $\alpha$-pinene and camphene (e.g., Figure 5), isothermal GC programming (at $180^{\circ} \mathrm{C}$ and 20 psi He pressure) was used.

FT-ICR MS. All GC/FT-ICR MS experiments were performed on a differentially pumped IonSpec 7-T

Table 1. Mass measurement accuracy and peak assignments for the observed peaks from GC/FT-ICR MS headspace analysis of camphene in heterodyne MS data acquisition mode

\begin{tabular}{lcccccc}
\hline Measured $m / z$ & Exact mass & $\begin{array}{c}\text { MMA } \\
(\mathrm{ppm})\end{array}$ & Elemental composition & Rel. Int. & R.P. m/ $\Delta \mathrm{m}_{50 \%}$ & Peak label in Fig. 5 \\
\hline \hline 135.1168 & 135.11683 & -0.22 & $\mathrm{C}_{10} \mathrm{H}_{15}{ }^{+}$ & 28.4 & 166000 & $\mathrm{x}$ \\
136.1203 & 136.12018 & 0.88 & ${ }^{13} \mathrm{C}_{1} \mathrm{C}_{9} \mathrm{H}_{15}{ }^{+}$ & 3.27 & 161800 & 1 \\
136.1247 & 136.12465 & 0.37 & $\mathrm{C}_{10} \mathrm{H}_{16}{ }^{+{ }^{+}}$ & 54.0 & 147900 & 2 \\
137.1280 & 137.12801 & -0.07 & ${ }^{13} \mathrm{C}_{1} \mathrm{C}_{9} \mathrm{H}_{16}{ }^{+{ }^{+}}$ & 6.4 & 185700 & 3 \\
137.1325 & 137.13248 & 0.15 & $\mathrm{C}_{10} \mathrm{H}_{17}{ }^{+}$ & 100.0 & 152300 & 4 \\
138.1359 & 138.13583 & 0.51 & ${ }^{13} \mathrm{C}_{1} \mathrm{C}_{9} \mathrm{H}_{17}{ }^{+}$ & 11.4 & 161800 & $y$ \\
& & $\langle|0.36|\rangle$ & & & & \\
\hline
\end{tabular}




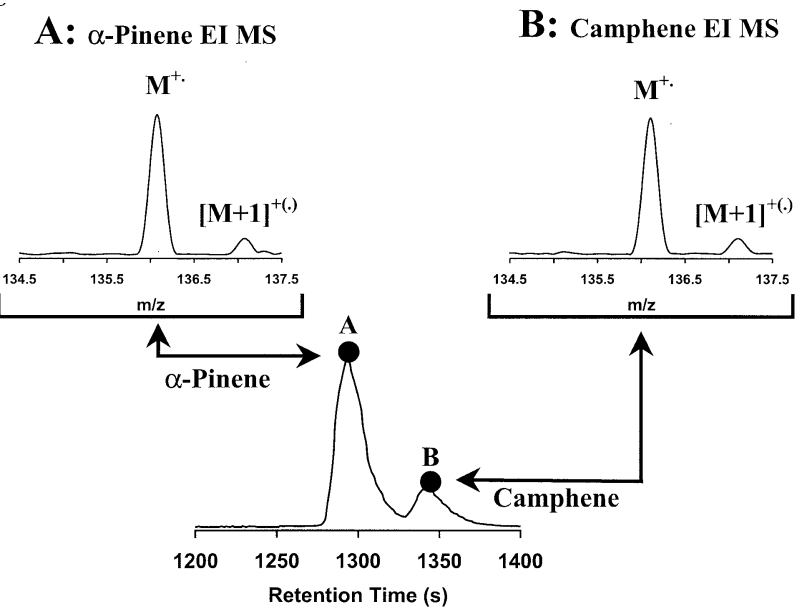

Figure 3. Bottom: The TIC from the GC/FT-ICR MS of authentic $\alpha$-pinene and camphene from $250 \mu \mathrm{L}$ of headspace that was withdrawn from a septum-sealed $40-\mathrm{mL}$ vial containing $\sim 50 \mathrm{mg}$ (sufficient to maintain saturated headspace) each of $\alpha$-pinene and camphene. The upper left and upper right panels show mass spectra of $\alpha$-pinene and camphene corresponding to TIC positions labeled A and B, respectively. The reaction delay for SCI was $\sim 5$ ms (see text for additional details).

FT-ICR mass spectrometer (Lake Forest, CA). Experiments were performed only in internal ion generation (24 eV EI) PC/GC/FT-ICR MS configuration [11]. The outer and inner trapping voltages for low resolution experiments (Figures 2, 3, and 4) were kept at 22.5 and $5 \mathrm{~V}$, respectively. For the high resolution mass spectral acquisition in the heterodyne mode (Figure 5 and Table 1 ), outer and inner trapping voltages were reduced to 3.0 and $0.5 \mathrm{~V}$, respectively. To investigate self-chemical ionization characteristics of terpene isomers, controlled ion-molecule reactions were performed on a different

\section{A: $\alpha$-Pinene EI/SCI MS}

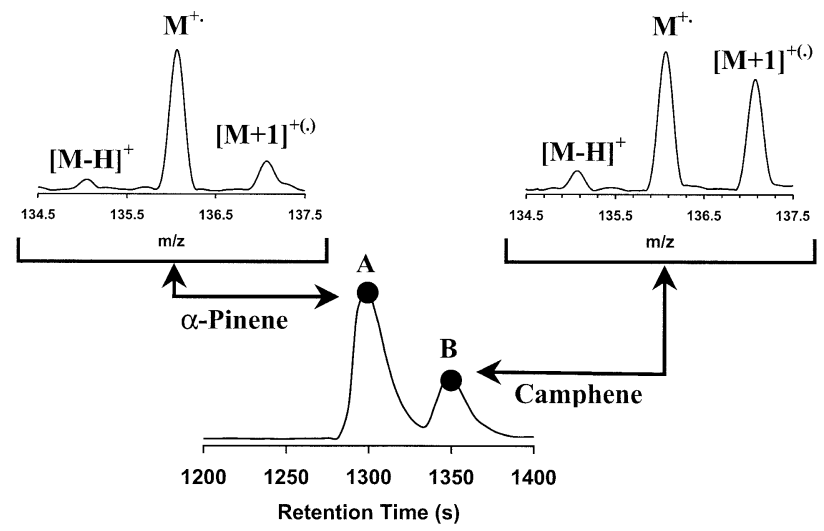

Figure 4. Bottom: The TIC from the GC/FT-ICR MS of authentic $\alpha$-pinene and camphene from $250 \mu \mathrm{L}$ of headspace that was withdrawn from a septum sealed $40-\mathrm{mL}$ vial containing $\sim 50 \mathrm{mg}$ (sufficient to maintain saturated headspace) each of $\alpha$-pinene and camphene. The upper left and upper right panels show mass spectra of $\alpha$-pinene and camphene corresponding to TIC positions labeled A and B, respectively. The reaction delay for SCI was $\sim 4400 \mathrm{~ms}$ (see text for additional details). (c)

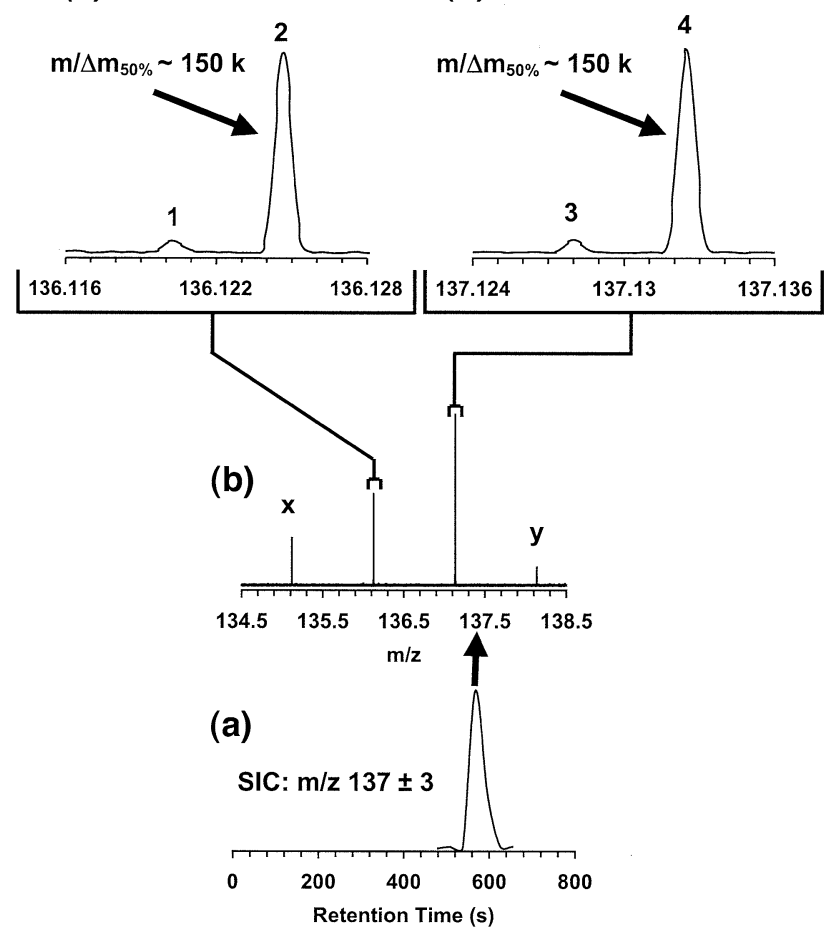

Figure 5. The GC/FT-ICR MS of authentic camphene from $30 \mu \mathrm{L}$ of headspace that was withdrawn from a septum-sealed $40-\mathrm{mL}$ vial containing $\sim 50 \mathrm{mg}$ (sufficient to maintain saturated headspace, SVP $\sim 2.6$ torr) camphene. Panel (a bottom) shows a narrow mass range $(\mathrm{m} / \mathrm{z} 137 \pm 3)$ chromatogram for the GC retention time of 8-12 min. Panel (b) shows high resolution mass spectrum $(\mathrm{m} / \mathrm{z}$ 134.5 to 138.5 ) used to generate the GC chromatogram shown in Panel (a). Top left (c) and right (d) insets show expanded regions $(\mathrm{m} / z 0.012$ mass range) of the mass spectra at $\mathrm{m} / \mathrm{z} \sim 136.1247$ and $\sim 137.1325$, respectively. Table 1 contains summary of the mass spectral data (e.g., mass resolving power and mass measurement accuracy) for Figure 5. Data acquisition parameters are provided in the Experimental section.

FT-ICR MS system. This system shared a common $7 \mathrm{~T}$ magnet with the GC/FT-ICR and was equipped with internal electron impact ionization source as well as a pulsed-leak valve sample introduction system [48].

FT-ICR MS Data Processing. The chromatogram and mass spectral $\mathrm{x}, \mathrm{y}$ coordinates were imported into the Microsoft Excel spreadsheets as ASCII delineated data [11]. The original time-domain transient signals contained $128 \mathrm{k}$ (Figure 2), $32 \mathrm{k}$ (Figures 3 and 4 ) and $1024 \mathrm{k}$ (Figure 5) data points. Figures 2, 3, 4, and 5 were acquired under broadband (16 $\mathrm{MHz}$ analog-to-digital converter $\{\mathrm{ADC}\}$ rate) and heterodyne (2 $\mathrm{MHz}$ analogto-digital converter $\{\mathrm{ADC}\}$ rate) modes, respectively. To display the mass spectra in Microsoft Excel graphs (as shown in Figures 2, 3, and 4), the fast Fourier transform (FFT) parameters were modified to transform only 32 kword of the acquired data with one zero fill. To generate the chromatograms in Microsoft Excel graphs (as shown in Figures 2, 3, and 4), the fast Fourier transform (FFT) parameters were modified to transform the acquired data without any zero fill. 


\section{Results and Discussion}

In this section, we show the GC and mass spectral data that we obtained from the analysis of the headspace from pine tree (Picea rubens) twig samples and commercially available authentic samples ( $\alpha$-pinene, camphene, limonene, and camphor). Both the GC retention times and mass spectra of commercially available terpene samples confirmed the original assignments of the unknown volatile organic compounds. We demonstrate better than one ppm mass measurement accuracy for highly resolved terpene pseudomolecular ions in GC/MS experiments. We also show that in addition to the GC retention times, gas-phase ion-molecule reactions can be used to differentiate structural isomers in real time.

\section{Emissions from the Pine Twigs}

Figure 2 shows the total-ion chromatogram (TIC) from a $100 \mathrm{~mL}$ sample of headspace volume surrounding pine tree (Picea rubens) twigs at room temperature, in a large volume $(\sim 2 \mathrm{~L})$ sealed ZipLoc bag, $\sim 24 \mathrm{~h}$ after specimen collection. Insets a to e show the mass spectra for TIC peaks labeled a to e, respectively. Accurate mass measurements revealed that the peaks in the mass spectra displayed in insets $b$ to e were all hydrocarbons in composition. Based on mass measurement accuracy, a cursory mass spectral pattern analysis and using the NIST online EI mass spectral database, we were able to identify the VOC species as $\alpha$-pinene, camphene, limonene, terpinilene, and camphor, in order of elution time (peaks labeled a to e in Figure 2). These assignments were later confirmed using authentic samples. The molecular structures of the identified VOCs are shown above the corresponding mass spectra. Samples analyzed $40 \mathrm{~min}, 20 \mathrm{~h}$, and $24 \mathrm{~h}$ after specimen collection did not show major temporal variations in relative VOC profiles.

Historically, GC characterization of terpene derivatives and other naturally occurring volatile compounds has played an important role in commercial activities, such as perfume formulation [49]. Solvent extraction analysis results $[22,25,26,30,31]$ may not reflect the actual quantitative or qualitative composition of the VOCs emitted into the gas-phase. Consequently, direct gas-phase sampling methods such as PC/GC/FT-ICR MS are preferable. For example, in Figure 2, at the relatively short GC retention time ( $R T \cong 490 \mathrm{~s}$ ), a peak corresponding to acetone is observed. Our method blanks showed no acetone signal, and confirmed that the acetone is, in fact, emitted from the pine twig samples. Although acetone is difficult to detect using conventional and destructive extraction methods [24], it has been shown to be present in VOC emissions from various trees [50, 51].

Based on accurate mass measurements and observed fragmentation patterns, the TIC peaks labeled a to d (in Figure 2) were ascribed to terpene isomers with the empirical formula, $\mathrm{C}_{10} \mathrm{H}_{16}$, and that of TIC Peak e to camphor, $\mathrm{C}_{10} \mathrm{H}_{16} \mathrm{O}$. The small GC peak at $\mathrm{RT} \cong 240 \mathrm{~s}$ is due $\mathrm{CO}_{2}$ previously trapped in the PC. The mass spectrum shown for Peak e (camphor) in the TIC in Figure 2, has been corrected for the continuous residual background. The heat generated from electron filament can enhance outgassing of the ICR cell components and contribute to the background over time. Using an FT-ICR mass calibration table generated $\sim 1$ month prior to the acquisition of the tree VOC data reported herein, the mean absolute mass measurement accuracy for the 8 major peaks (mass spectral peaks with $\mathrm{S} / \mathrm{N}>$ $\sim 20$ present in insets 1 to 3 of Figure 2) was $<5.0 \mathrm{ppm}$ $(2.4 \pm 0.9 \mathrm{ppm}$ at the $95 \%$ confidence level for $\mathrm{n}=24$, based on absolute errors using $\mathrm{m} / \mathrm{z}$ values from three mass spectra). The authentic samples were used to generate new mass calibration tables and improve mass measurement accuracy. For example, in heterodyne mode, Figure 5 (Table 1), the average mass measurement error was $0.4 \pm 0.2$ at the $95 \%$ confidence level, with $n=6$.

\section{Authentic Sample Analysis and Isomer Differentiation}

Commercially available authentic samples were purchased and analyzed to confirm the assigned identities of the unknown analytes. Terpinolene (Peak d in Figure 2) was not readily available to confirm our initial assignment; however, the pattern observed in Figure 2d closely matches terpinolene's mass spectrum contained in the NIST's online database (http://webbook.nist. gov/chemistry/). Comparisons between the GC retention times as well as observed mass spectral fragmentation patterns of the unknows and authentic samples confirm the correct assignments of the Peaks a, b, c and e in Figure 2 as $\alpha$-pinene, camphene, limonene, and camphor, respectively. These four volatile compounds were previously identified as the major components of the leaf oils from Picea species [21].

Figure 3 (bottom) shows a TIC segment from PC/ GC/FT-ICR MS analysis of the authentic $\alpha$-pinene and camphene isomers (from RT $=1200$ to $1400 \mathrm{~s}$ ). To acquire the data in Figure 3, $250 \mu \mathrm{L}$ of headspace was withdrawn from a septum sealed $40-\mathrm{mL}$ vial that contained $\sim 50 \mathrm{mg}$ each of $\alpha$-pinene and camphene. The 50 $\mathrm{mg}$ was in large excess of the amount required to maintain saturated vapor pressures (SVPs) of camphene $\left(\mathrm{SVP}=2.6\right.$ torr) and $\alpha$-pinene (SVP $=5.0$ torr) at $25^{\circ} \mathrm{C}$ [52]. The headspace sample was manually injected onto the GC for FT-ICR MS analysis. To correct for the PC transfer line delay times, additional experiments for all four authentic $\alpha$-pinene, camphene, limonene, and camphor samples were performed (data not shown). The GC retention times shown in Figures 3 and 4 were corrected for the PC/GC transfer line $(\sim 2 \mathrm{~m}$ in total length) delay time $(\sim 20 \mathrm{~s}$, the time required for samples to travel from module 3 in PC to GC injection port, as 
measured experimentally). Comparisons between the GC retention times as well as the observed mass spectral fragmentation patterns of unknowns and authentic samples confirmed the correct assignments of the peaks a, b, c and e in Figure 2 as $\alpha$-pinene, camphene, limonene, and camphor, respectively.

The GC peaks in Figure 3 (RT 1280-1325 s and $\mathrm{RT} \sim 1325-1370 \mathrm{~s}$ ) are made up of about ten averaged mass spectra (viz., total of about five hundred mass spectra acquired across each GC peak). In other words, each point (e.g., Points A or B) on the GC plot of Figure 3 represents an average of fifty mass spectra. The observed GC baseline peak widths for $\alpha$-pinene and camphene were $\sim 45 \mathrm{~s}$ each. The longitudinal diffusion and GC peak broadening occurs within the 2 meter $(0.5$ $\mathrm{mm}$ i.d.) transfer line due to reduced mass flow rate after the jet separator. The current GC software requires a $1 \mathrm{~s}$ delay before acquiring the next averaged mass spectrum. The duty cycle for each mass spectrum was about $60 \mathrm{~ms}$, and the electron beam was on for $45 \mathrm{~ms}$. To reduce interferences from the metastable decay and/or ion-molecule reactions inside the ICR cell, we used short duty cycles (i.e., $60 \mathrm{~ms}$ in this case). Prior to ion detection, there was only $\sim 5 \mathrm{~ms}$ reaction delay event after the end of the ionization period (the ion excitation and detection events require $\sim 10 \mathrm{~ms}$ ). Hence, ionmolecule reactions were insignificant and did not alter the observed mass spectral appearances. The appearance of the GC/FT-ICR mass spectra (e.g., ethanol, acetone, hexane, benzene, toluene, $\mathrm{m}$-xylene, $\alpha$-pinene, camphene, limonene, and camphor) at such short delay times resembled conventional $70 \mathrm{eV}$ mass spectra and, therefore, NIST (http://webbook.nist.gov/chemistry/) or other conventional mass spectral library searches could be used to identify unknown analytes.

The molecular ion regions for $\alpha$-pinene and camphene $(m / z 134.5-137.5)$ that correspond to mass spectra of Points A and B in the TIC of Figure 3 are shown in Figure $3 a$ and $b$, respectively. The molecular ion regions for both $\alpha$-pinene and camphene are virtually identical. The relative abundance of $\mathrm{M}^{+}$and $[\mathrm{M}+1]^{+}$peaks of the $\alpha$-pinene and camphene suggest that for the $60 \mathrm{~ms}$ duty cycle the carbon thirteen isotopomer $\left[{ }^{12} \mathrm{C}_{9}^{13} \mathrm{C}_{1} \mathrm{H}_{16}\right]^{\cdot+}$ ions $\left({ }^{13} \mathrm{C}_{1}\right.$ of $\mathrm{M}^{++}$at $\left.m / z=137.128\right)$ are the major components of the $[\mathrm{M}+1]^{+}$peaks at $m / z 137$. Potential $[\mathrm{M}+\mathrm{H}]^{+}$ions from SCI $\left({ }^{12} \mathrm{C}_{10} \mathrm{H}_{17}^{+}\right.$at $m / z=$ 137.132) do not significantly contribute to the intensity of the peak at $m / z \sim 137$.

Figure 4 shows a TIC segment from PC/GC/FT-ICR MS analysis of the authentic $\alpha$-pinene and camphene samples (from RT $=1200$ to $1400 \mathrm{~s}$ ). The injected headspace volume (from the $40-\mathrm{mL}$ vial containing sufficient $\alpha$-pinene and camphene to maintain saturated vapor pressures) and GC variable parameters were identical in Figures 3 and 4. However, the mass spectral data acquisition parameters for Figures 3 and 4 were different. Specifically, each point on the GC plot of Figure 4 represents only a single mass spectrum (vis-àvis average of fifty mass spectra in Figure 3). For the mass spectral SCI-based differentiation of $\alpha$-pinene and camphene, we increased the time required to acquire each mass spectrum from $60 \mathrm{~ms}$ in Figure 3 to $5000 \mathrm{~ms}$ in Figure 4. In addition, we increased the ionization event (electron beam on) from $45 \mathrm{~ms}$ (in Figure 3) to 600 $\mathrm{ms}$ in (Figure 4 ). The increased reaction delay from $\sim 5$ ms (in Figure 3) to $\sim 4400 \mathrm{~ms}$ (in Figure 4 ) allowed for ion-molecule reactions to take place inside the ICR cell.

The molecular ion regions for $\alpha$-pinene and camphene $(m / z 134.5-137.5)$ that correspond to mass spectra of Points A and B in the TIC of Figure 4 are shown in Figure $4 \mathrm{a}$ and $\mathrm{b}$, respectively. The GC TIC for Figures 3 and 4 are similar. Conversely, the molecular ion regions for $\alpha$-pinene and camphene in Figures 3 and 4 are different. For example, at the longer reaction delay time of $\sim 4400 \mathrm{~ms}$ (in Figure 4 ), new peaks at $m / z \sim 135$ that correspond to products of hydride abstraction from $\alpha$-pinene and camphene were observed. More significantly, protonated molecules from SCI at $\mathrm{m} / \mathrm{z} \sim 137$ were formed. The relative ratios of $\mathrm{M}^{+}$to $[\mathrm{M}+1]^{+}$ peaks for the $\alpha$-pinene (Figure $4 \mathrm{a}$ ) and camphene (Figure $4 b)$ were significantly different. The superscript $(\cdot)+$ symbols in the panels of Figures 3 and 4 indicate that the mass spectral peaks at $m / z \sim 137$, for both isomers, may contain ${ }^{13} \mathrm{C}_{1} \mathrm{M}^{++}$or $\left[{ }^{12} \mathrm{C}_{9}^{13} \mathrm{C}_{1} \mathrm{H}_{16}\right]^{++}(m / z=137.128)$ as well as $[\mathrm{M}+\mathrm{H}]^{+} \mathrm{SCI}$ product ${ }^{12} \mathrm{C}_{10} \mathrm{H}_{17}^{+}(\mathrm{m} / \mathrm{z}=$ $137.132)$ ions. Figure $5 d$, contains high resolution mass spectrum of the camphene molecular ion region, acquired under different experimental conditions where the isotopomers were fully resolved (mass resolving power, $\sim 150,000$, Table 1).

A comparison between Figure $4 \mathrm{a}$ and $\mathrm{b}$ indicates that the relative abundance of the protonated camphene is higher than that of the $\alpha$-pinene isomer. During the SCI events, the relative abundance of the camphene GC peak was lower than that of $\alpha$-pinene GC peak. Assuming similar ionization cross sections for the two isomers, we conclude that the relative partial pressure of $\alpha$-pinene $\left(\mathrm{IE}_{\alpha \text {-pinene }}=8.07 \mathrm{eV}\right)$ during the SCI inside the ICR cell (TIC Point A in Figure 4) was at least two-fold higher than camphene's ( $\mathrm{IE}_{\text {camphene }} \leq 8.86 \mathrm{eV}$ ) (see http:/ /webbook.nist.gov) partial pressure (TIC Point B in Figure 4). Compared to camphene, even at a higher $\alpha$-pinene relative partial pressure, the SCI of $\alpha$-pinene in Figure 4 was negligible and the peak for $[\mathrm{M}+1]^{+}$in Figure $4 \mathrm{~b}$ was higher than the corresponding peak in Figure 4a. The relative heights of the $\mathrm{M}^{\cdot+}$ and $[\mathrm{M}+1]^{+}$ peaks in Figure $4 \mathrm{a}$ and $\mathrm{b}$ can be used to differentiate $\alpha$-pinene and camphene isomers. Further details on $\alpha$-pinene and camphene SCI have been presented at the 2003 ASMS conference [53] and is also the subject matter of a forthcoming article [54]. A brief discussion is provided in the following paragraph.

Controlled ion-molecule experiments, using a pulsed-leak valve sample introduction system [48], were performed to determine the factors giving rise to the different SCI patterns of camphene and $\alpha$-pinene. Preliminary results were presented [55] and full details and results will be published in the near future. Briefly, 
major ions produced in the 24-eV EI of camphene or $\alpha$-pinene (viz., $m / z, 91,93,107,121,136)$ were individually SWIFT isolated and then allowed to react either unimolecularly or by SCI for specified reaction delays. It was observed that only camphene molecular ions had a major long-lived metastable component $(\sim 50 \%, \tau \sim 30$ $\mathrm{s}$, major product ions at $\mathrm{m} / \mathrm{z} 92,94$, and 121). These excited ions gave rise to the SCI product ion at $m / z 137$. No metastable $\alpha$-pinene molecular ions were observed under our experimental conditions (time between EI and detection events varied from $\sim 450 \mathrm{~ms}$ to $500 \mathrm{~s}$ ). The minimum time for ion isolation (due to high pressure and SWIFT time requirement) was $450 \mathrm{~ms}$ after the ionization event. The precursor $\alpha$-pinene and "cold" camphene molecular ions, as well as their major fragment ions, yielded an insignificant amount of $\mathrm{m} / \mathrm{z} 137$ by SCI.

Proton transfer bracketing reactions (data not shown here) suggest that the proton affinities for the two terpenes are similar and approximately $\sim 210 \mathrm{kcal} /$ mole. A density functional theory (DFT) calculation by Ebmeyer places $\alpha$-pinene proton affinity less than one $\mathrm{kcal} /$ mole above that of camphene [56]. Proton affinities alone are not sufficient to determine the thermochemistry of a proton-transfer reaction. For any proton transfer reaction, acidities of the ions and basicities of the neutrals will determine the overall free energy change. The proton affinities of $\alpha$-pinene and camphene are similar. Hence, acidities of the $\alpha$-pinene and camphene molecular ions could be different; similarly, acidities of their fragment ions, such as $m / z 93$ from camphene compared with $m / z 93$ from $\alpha$-pinene, could be different. We observed that isolated "cold" camphene molecular ions did not yield measurable SCI. Therefore, considering the importance of kinetic and thermodynamic controlling factors, acidity of the excited $\mathrm{M}^{+}$camphene ions may be an important controlling factor for camphene SCI.

Figure 5 shows a GC selected ion chromatogram (SIC) and FT-ICR high resolution mass spectrum obtained from analysis of $30 \mu \mathrm{L}$ of headspace taken from a septum-capped $40-\mathrm{mL}$ vial containing a sufficient camphene sample to maintain saturated vapor pressure. Using camphene saturated vapor pressures at various temperatures [52] and integrated form of Clausius-Clapeyron equation (i.e., $\ln \mathrm{P}$ versus $1 / \mathrm{T}$ ), the vapor pressure of camphene at the laboratory temperature of $25^{\circ} \mathrm{C}$ was calculated to be $\sim 2.6$ torr (i.e., $\sim 4.0$ nmole injection onto GC column from the $30 \mu \mathrm{L}$ camphene headspace sample). The data acquisition was started 8 min after the GC injection event to reduce the computer data file size. A 500-ms GC effluent segment was pulsed into the ICR cell approximately every $29 \mathrm{~s}$. The GC peak was $\sim 25 \mathrm{~s}$ wide at half height (determined from separate experiments). The displayed insets were all constructed (in Microsoft Excel) from mass spectral data acquired in heterodyne mode $(m / z 136 \pm 10)$. The $1024 \mathrm{k}$ time domain data were fast Fourier transformed (Blackman window with two zero fills) to frequency domain data using IonSpec furnished software. The resulting frequency domain data were converted to ASCII mass spectral data and imported into Microsoft Excel. Advanced curve fittings were not used in Microsoft Excel and only line connectors were used to join the raw data points. IonSpec software utilizes more advanced curve fitting functions to display mass spectral peaks. These different curve fitting methods induce small differences in the derived mass spectral parameters (e.g., peak centroid, peak width). In Table 1, we have listed the more conservative mass resolution values generated by IonSpec software.

Figure 5a (bottom) shows the SIC for $m / z 137 \pm 3$. The SIC peak contains only 3 points. A duty cycle of $\sim 29 \mathrm{~s}$ was used to reduce the pressure inside the ICR cell to below $1 \times 10^{-9}$ torr (as indicated on a remote ion gauge display) during the excite and detect events. Figure $5 \mathrm{~b}$ (middle) shows the mass spectrum corresponding to the SIC peak maximum. Figure $5 \mathrm{c}$ and $\mathrm{d}$ (top two insets), show expanded regions $(\mathrm{m} / \mathrm{z} 0.012$ mass range) of the peaks at $m / z \sim 136.1247$ and $\sim 137.1325$ from Figure 5b. Peak at $m / z$ 136.1203, (labeled as 1 in Figure $5 \mathrm{c}$ ), is the ${ }^{13} \mathrm{C}_{1}$ isotope of the camphene $[\mathrm{M}-\mathrm{H}]^{+}$ion and peak at $\mathrm{m} / \mathrm{z}$ 136.1247, (labeled as 2 in Figure 5c), corresponds to the camphene molecular ion $\left(\mathrm{M}^{+}\right)$. Peak at $\mathrm{m} / \mathrm{z}$ 137.1280, (labeled as 3 in Figure $5 \mathrm{~d}$ ) is the ${ }^{13} \mathrm{C}_{1}$ isotope of $\mathrm{M}^{+}$, whereas peak at $m / z$ 137.1325, (labeled as 4 in Figure 5d), corresponds to the protonated camphene, $[\mathrm{M}+\mathrm{H}]^{+}$. The mass resolving powers $\left(\mathrm{m} / \Delta \mathrm{m}_{50 \%}\right)$ for all observed peaks in Figure 5 were in the range of $140,000-185,000$ (Table 1). The relative abundance of ${ }^{13} \mathrm{C}_{1}$ peaks is in good agreement with natural abundance of carbon isotopes. The method detection limit (at S/N of 3) for high resolution GC/FTICR of camphene at $\mathrm{m} / \mathrm{z} 137(\mathrm{~S} / \mathrm{N}=150$ for an oncolumn injection of $4.0 \mathrm{nmol}$ of camphene) is estimated to be $<2$ pmol. A summary of the mass spectral data is provided in Table 1. Columns 1-7 contain experimentally measured masses, exact masses, mass measurement accuracy, elemental compositions, relative ion intensities, mass resolving power, and peak labels used in Figure 5, respectively. The mean absolute mass measurement error of $<0.36>$ ppm for high resolution GC/FT-ICR MS data (Figure 5) is given at the bottom of the third column in Table 1.

For the isomer differentiation method presented here, we have used the analyte molecules as the chemical reagent gases for SCI. However, other reagents (not investigated in the present study) can be used to enhance the selectivity of this method and extend its utility to other classes of chemicals. In addition to protonation, hydride abstraction, fragmentation, dimer formation, and other ion-molecule reactions may be used for isomer differentiation. Although we only show the mass spectral comparison between Points A and B (presumably the high-pressure regions of the GC TIC) [11], it should be noted that enhanced SCI is observed for camphene across the GC TIC peak in Figure 4. 


\section{Conclusions}

The successful coupling of a PC to GC/FT-ICR MS was described. Ion-molecule reactions such as SCI were utilized to add an additional dimension to modern GC/MS analysis protocols. The PC/GC/FT-ICR MS requires minimal sample preparation and provides remarkable analytical performance characteristics such as high mass measurement accuracy and high mass resolving power.

\section{Acknowledgments}

The authors gratefully acknowledge support from the Defense Advanced Research Projects Agency (N65236-98-1-5415) and the National Science Foundation (CHE-0228971). They express their gratitude to Professor Barbara J. W. Cole for her assistance in the literature survey of terpene chemistry. They also appreciate the helpful comments of the editor and the reviewers.

\section{References}

1. Robinson, A. EHC 155: Biomarkers and Risk Assessment: Concepts and Principles; World Health Organization: Geneva, Switzerland, 1993, pp 1-82.

2. Smith, D.; Spanel, P.; Davies, S. Trace Gas in Breath of Healthy Volunteers when Fasting and After a Protein-Calorie Meal: A Preliminary Study. J. Appl. Physiol. 1999, 87, 1584-1588.

3. Yu, K.; Hamilton-Kemp, T. R.; Archbold, D. D.; Collins, R. W.; Newman, M. C. Volatile Compounds from Escherichia coli O157:H7 and Their Absorption by Strawberry Fruit. J. Agric. Food Chem. 2000, 48, 413-417.

4. Demirev, P. A.; Lin, J. S.; Pineda, F. J.; Fenselau, C. Bioinformatics and Mass Spectrometry for Microorganism Identification: Proteome-Wide Post-Translational Modifications and Database Search Algorithms for Characterization of Intact. $H$. Pylori. Anal. Chem. 2001, 73, 4566-4573.

5. Lee, M. L.; Smith, D. L.; Freeman, L. R. High-Resolution Gas Chromatographic Profiles of Volatile Organic Compounds Produced by Microorganisms at Refrigerated Temperatures. Appl. Environ. Microbiol. 1979, 37, 85-90.

6. Takayasu, T.; Ohshima, T.; Kondo, T.; Sato, Y. Intratracheal Gas Analysis for Volatile Substances by Gas Chromatography/Mass Spectrometry Application to Forensic Autopsies. J. Forensic Sci. 2001, 46, 98-104.

7. Fievre, A.; Solouki, T.; Marshall, A. G.; Cooper, W. T. HighResolution Fourier Transform Ion Cyclotron Resonance Mass Spectrometry of Humic and Fulvic Acids by Laser Desorption/Ionization and Electrospray Ionization. Ener. Fuels 1997, 11, 554-560.

8. Rodgers, R. P.; White, F. M.; Hendrickson, C. L.; Marshall, A. G.; Andersen, K. V. Resolution, Elemental Composition, and Simultaneous Monitoring by Fourier Transform Ion Cyclotron Resonance Mass Spectrometry of Organosulfur Species Before and After Diesel Fuel Processing. Anal. Chem. 1998, 70, 4743-4750.

9. Craig, A. G.; Speir, J. P.; Rosamilia, S.; Fursey, V.; Lirazan, M. B. $[\mathrm{M}+\mathrm{Fe}-5 \mathrm{H}]^{2-}$ Peptide Ion Composition Verified by Fourier Transform Mass Spectrometry Accurate Mass and Tandem Mass Spectrometry Analyses. J. Am. Soc. Mass Spectrom. 2000, 11, 83-87.

10. Beynon, J. H. Qualitative Analysis of Organic Compounds by Mass Spectrometry. Nature 1954, 174, 735-737.

11. Szulejko, J. E.; Solouki, T. Potential Analytical Applications of Interfacing a GC to an FT-ICR MS: Fingerprinting Complex Sample Matrixes. Anal. Chem. 2002, 74, 3434-3442.
12. McFadden, W. Techniques of Combined Gas Chromatography/Mass Spectrometry; Wiley: New York, 1973, pp 1-482.

13. Masselon, C.; Tolmachev, A. V.; Anderson, G. A.; Harkewicz, R.; Smith, R. D. Mass Measurement Errors Caused by Local Frequency Perturbations in FTICR Mass Spectrometry. J. Am. Soc. Mass Spectrom. 2002, 13, 99-106.

14. Burton, R. D.; Matuszak, K. P.; Watson, C. H.; Eyler, J. R. Exact Mass Measurement Using a 7 Tesla Fourier Transform Ion Cyclotron Resonance Mass Spectrometer in a Good Laboratory Practices-Regulated Environment. J. Am. Soc. Mass Spectrom. 1999, 10, 1291-1297.

15. Hughey, C. A.; Hendrickson, C. L.; Rodgers, R. P.; Marshall, A. G.; Qian, K. Kenrdrick Mass Defect Spectrum: A Compact Visual Analysis for Ultrahigh-Resolution Broadband Mass Spectra. Anal. Chem. 2001, 73, 4676-4681.

16. He, F.; Hendrickson, C. L.; Marshall, A. G. Baseline Mass Resolution of Peptide Isobars: A Record for Molecular Mass Resolution. Anal. Chem. 2001, 73, 647-650.

17. Ragunathan, N.; Krock, K. A.; Klawun, C.; Sasaki, T. A.; Wilkins, C. L. Gas Chromatography with Spectroscopic Detectors. J. Chromatogr. A 1999, 856, 349-397.

18. Harris, C. M. Waiting to Exhale: Meeting News from the Federation of Analytical Chemistry and Spectroscopy Societies (FACSS) Meeting PC/GC/FT-ICR MS for Exhaled Breath Analysis by Touradj Solouki, Jan Szulejko. Anal. Chem. 2001, 73, 658A-659A.

19. Ochiai, N.; Takino, M.; Daishima, S.; Cardin, D. B. Analysis of Volatile Sulphur Compounds in Breath by Gas Chromatography-Mass Spectrometry Using a Three-Stage Cryogenic Trapping Preconcentration System. J. Chromatogr. B 2001, 762, 67-75.

20. Jacoby, C. B.; Remple, D. L.; Gross, M. L. A Cold Trap/Pulsed Valve GC/FTMS Interface: Ultra-Trace Analysis. Proceeding of the 38th ASMS Conference on Mass Spectrometry and Allied Topics; Tucson, AZ, 1990, pp 840-841.

21. Yatagai, M.; Sato, T. Terpenes of Leaf Oils from Conifers. Biochem. Syst. Ecol. 1986, 14, 469-478.

22. Roussis, V.; Petrakis, P. V.; Ortiz, A.; Mazomenos, B. E. Volatile Constituents of Needles of Five Pinus Species Grown in Greece. Phytochem. 1995, 39, 357-361.

23. Hanover, J. W. Environmental Variation in the Monoterpenes of. Pinus monticola Dougl. Phytochem. 1966, 1966, 713-717.

24. Wilkinson, R. C.; Hanover, J. W. Geographical Variation in the Monoterpene Composition of Red Spruce. Phytochem. 1972, 11, 2007-2010.

25. Jüttner, F. Changes of Monoterpene Concentration in Needles of Pollution-Injured Picea abies Exhibiting Montane Yellowing. Physiol. Plant 1988, 72, 48-55.

26. Schulten, H. R.; Simmleit, N.; Rump, H. H. Forest Damage: Characterization of Spruce Needles by Pyrolysis Field Ionization Mass Spectrometry. Int. J. Environ. Anal. Chem. 1986, 27, 241-264.

27. Paré, P. W.; Tumlinson, J. H. Plant Volatile Signals in Response to Herbivore Feeding. Florida Entomol. 1996, 79, 93-103.

28. Winer, A. M.; Atkinson, R.; James, N.; Pitts, J. Gaseous Nitrate Radical: Possible Nighttime Atmospheric Sink for Biogenic Organic Compounds. Science 1984, 224, 156-158.

29. Rudloff, E. V. Seasonal Variation in the Composition of the Volatile Oil of the Leaves, Buds, and Twigs of White Spruce (Picea glauca). Can. J. Bot. 1972, 50, 1595.

30. Muzika, R. M.; Campbell, C. L.; Hanover, J. W.; Smith, A. L. Comparison of Techniques for Extracting Volatile Compounds from Conifer Needles. J. Chem. Ecol. 1990, 16, 2713-2723.

31. Dormont, L.; Roques, A.; Malosse, C. Cone and Foliage Volatiles Emitted by Pinus cembra and Some Related Conifer Species. Phytochem. 1998, 49, 1269-1277. 
32. Kim, J.-C. Factors Controlling Natural VOC Emissions in a Southeastern U.S. Pine Forest. Atmosph. Environ. 2001, 35, 3279-3292.

33. Williams, E. R.; McLafferty, F. W. One Hundred Ninety Three-nm Laser Photoionization and Photodissociation for Isomer Differentiation in Fourier-Transform Mass Spectrometry. J. Am. Soc. Mass Spectrom. 1990, 1, 361-365.

34. Sack, T. M.; McCrery, D. A.; Gross, M. L. Gas Chromatography/Multiphoton Ionization Fourier Transform Mass Spectrometry. Anal. Chem. 1985, 57, 1290-1295.

35. Büker, H.-H.; Grützmacher, H.-F.; Crestoni, M. E.; Ricci, A. Proton Induced Methyl Group Shifts in Gaseous Xylenium Ions. Distinguishing Isomers by Gas-phase Titration. Int. J. Mass Spectrom. Ion Processes 1997, 160, 167-181.

36. Sharifi, M.; Einhorn, J. Isomeric Differentiation of Conjugated Diene Epoxides by Polar [4+2+] Diels-Alder Cycloaddition of Acylium Ions in an Ion Trap Mass Spectrometer. Int. J. Mass Spectrom. 1999, 190/191, 253-264.

37. Dolnikowski, G. G.; Gross, M. L. Isomer Differentiation in 7,12-Dimethylbenz $[\alpha]$ anthracene-Pyridine Adducts by Fast Atom Bombardment Tandem Mass Spectrometry. J. Am. Soc. Mass Spectrom. 1991, 2, 256-258.

38. Tao, W. A.; Wu, L.; Cooks, R. G. Differentiation and Quantitation of Isomeric Dipeptides by Low-energy Dissociation of Copper(II)-bound Complexes. J. Am. Soc. Mass Spectrom. 2001, 12, 490-496.

39. Waridel, P.; Wolfender, J.-L.; Ndjoko, K.; Hobby, K. R.; Major, H. J.; Hostettmann, K. Evaluation of Quadrupole Time-ofFlight Tandem Mass Spectrometry and Ion-trap MultipleStage Mass Spectrometry for the Differentiation of C-Glycosidic Flavonoid Isomers. J. Chromatogr. A 2001, 926, 29-41.

40. Mosi, A. A.; Cullen, W. R.; Eigendorf, G. K. Ion/Molecule Reactions of Perfluorotributylamine (FC43) Cations with Polycyclic Aromatic Hydrocarbons in a Quadrupole Ion Trap. Int. J. Mass Spectrom. 1999, 190/191, 195-207.

41. Miles, W. F.; Gurprasad, N. P.; Malis, G. P. Isomer-Specific Determination of Hexacholrodibenzo-p-Dioxins by Oxygen Negative Chemical Ionization Mass Spectrometry, Gas Chromatography, and High-Pressure Liquid Chromatography. Anal. Chem. 1985, 57, 1133-1138.

42. Crespín, M. A.; Cárdenas, S.; Gallego, M.; Valcárcel, M. Discrimination of Structural Isomers of Chlorinated Phenols in Waters Using Gas Chromatography-Mass Spectrometry in the Negative Chemical Ionization Mode. J. Chromatogr. A 1999, 830, 165-174.

43. Hayakawa, S.; Taguchi, K.; Kotani, R.; Arakawa, K.; Morishita, N. Discrimination of Isomers of Dichlorobenzene Using
Charge Inversion Mass Spectrometry. J. Mass Spectrom. Soc. Japan 2001, 49(219), 223.

44. Bulpitt, C.; Tsang, S. C. Detection and Differentiation of $\mathrm{C}_{4}$ Hydrocarbon Isomers Over the $\mathrm{Pd}-\mathrm{SnO}_{2}$ Compressed Powder Sensor. Sensors and Actuators. B: Chemical. 2000, 69, 100-107.

45. Gordon, A. G. The Taxonomy and Genetics of Picea rubens and Its Relationship to Picea mariana. Can. J. Bot. 1976, 54, 781-813.

46. Brooks, J. E.; Pierce, H. D.; Borden, J. H.; Lister, G. R. Seasonal Variation in Foliar and Bud Monoterpenes in Sitka Spruce. Can. J. Bot. 1987, 65, 1249-1252.

47. Sack, T. M.; Gross, M. L. Pulsed Valve Interface for Gas Chromatography/Fourier Transform Mass Spectrometry. Anal. Chem. 1983, 55, 2419-2421.

48. Solouki, T.; Fort, R.; Alomary, A.; Fattahi, A. Gas-Phase Hydrogen- Deuterium Exchange Reactions of a Model Peptide: FT-ICR and Computional Analyses of Metal Induced Conformational Mutations. J. Am. Soc. Mass Spectrom. 2001, 12, 1272-1285.

49. Dudley, B. Perfumery Chemicals from Pinene. Chem. Ind. 1964, 2, 263-267.

50. Janson, R.; deServes, C. Acetone and Monoterpene Emissions from the Boreal Forest in Northern Europe. Atmosph. Environ. 2001, 35, 4629-4637.

51. Goldstein, A. H.; Schade, G. W. Quantifying Biogenic and Anthropogenic Contributions to Acetone Mixing Ratios in a Rural Environment. Atmosph. Environ. 2000, 34, 4997-5006.

52. Lide, D. R.; Frederikse, H. P. R., Eds.; CRC Handbook of Chemistry and Physics, 77th ed. CRC Press Inc.: Baton Rouge, LA, 1996/1997; Sections 6-112.

53. Szulejko, J. E.; Bennett, J. B.; Graham, L. B.; Solouki, T. Kinetic Analysis of Metastable Decay and Ion-Molecule Reactions for Selected Terpene Isomers: Cold and Hot Ions. Proceedings of the 51st ASMS Conference on Mass Spectrometry and Allied Topics; Montreal, QC, Canada, 2003; p 2138.

54. Szulejko, J. E., Solouki, T. Self-Chemical Ionization of Camphene and $\alpha$-Pinene Isomers. Ionic Excited States, Acidities, Neutral Proton Affinities and Other Thermochemical Considerations, unpublished.

55. Bennett, J. B.; Graham, L. B.; Szulejko, J. E.; Solouki, T. Differentiation of Terpene Isomers Based on Unimolecular and Ion-Neutral Reactions. Proceedings of the 4th North American FT-ICR MS Conference; Marshall, CA, April, 2003.

56. Ebmeyer, F. Theoretical Investigations Towards an Understanding of the $\alpha$-Pinene/Camphene Rearrangement. J. Molec. Struct. (Theochem.) 2002, 582, 251-255. 